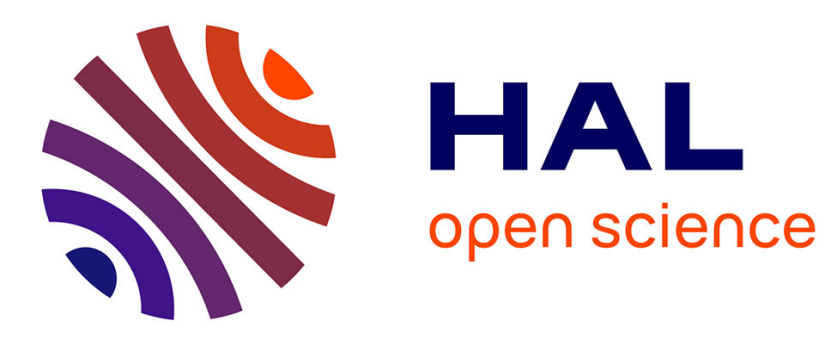

\title{
Incorporation et rôle des halogènes dans les silicates vitreux et fondus
}

Lucie Grousset, Éric Pili, Daniel Neuville

\section{To cite this version:}

Lucie Grousset, Éric Pili, Daniel Neuville. Incorporation et rôle des halogènes dans les silicates vitreux et fondus. Matériaux \& Techniques, 2015, 103 (4), 10.1051/mattech/2015040 . insu-01906100

\section{HAL Id: insu-01906100 \\ https://hal-insu.archives-ouvertes.fr/insu-01906100}

Submitted on 26 Oct 2018

HAL is a multi-disciplinary open access archive for the deposit and dissemination of scientific research documents, whether they are published or not. The documents may come from teaching and research institutions in France or abroad, or from public or private research centers.
L'archive ouverte pluridisciplinaire HAL, est destinée au dépôt et à la diffusion de documents scientifiques de niveau recherche, publiés ou non, émanant des établissements d'enseignement et de recherche français ou étrangers, des laboratoires publics ou privés. 


\title{
Incorporation et rôle des halogènes dans les silicates vitreux et fondus
}

\author{
Lucie Grousset $^{1}$, Eric Pili ${ }^{1,2}$ et Daniel R. Neuville ${ }^{1}$
}

\begin{abstract}
Reçu le 29 mai 2015, accepté le 27 juillet 2015
Résumé - Cet article propose une synthèse sur la solubilité, la spéciation et la diffusion des halogènes (fluor, chlore, brome et iode) dans les silicates fondus ainsi que leur effet sur la viscosité. Le fluor est l'halogène le plus soluble dans les silicates fondus. En se substituant à l'oxygène et en s'associant à l'aluminium, il favorise la dépolymérisation du réseau, ce qui se traduit par une baisse de la viscosité du verre. La solubilité du chlore est favorisée par la présence d'alcalins dans la composition du liquide. Le chlore s'associe aux cations modificateurs de réseau comme le calcium ou le sodium. Son effet sur la viscosité dépend de la composition chimique de la matrice vitreuse, notamment de la proportion d'alcalins et d'aluminium. La solubilité du brome est augmentée dans un liquide peralcalin riche en silice. La solubilité de l'iode peut atteindre $1 \%$ en masse dans un verre borosilicaté. L'iode s'associe alors aux ions sodium.
\end{abstract}

Mots clés : Silicates fondus / halogènes / solubilité / spéciation / viscosité

Abstract - Incorporation and role of halogens in silicate melts and glasses. This article provides a review on the solubility, speciation and diffusion of halogens (fluorine, chlorine, bromine and iodine) in molten silicates and their effect on the viscosity. Fluorine is the most soluble halogen in silicate melts. By replacing oxygen in tetraedra and by coordinating with aluminum, it triggers depolymerization of the glassy network, which decreases the viscosity of silicate melt. The solubility of chlorine is favored by the presence of alkali in the melt composition. Chlorine shows affinity for the network modifiers cations such as calcium or sodium. Its effect on viscosity depends on the chemical composition, especially the proportion of alkali elements and aluminum. The solubility of bromine increases in silica-rich and alkali-rich melt. The solubility of iodine can reach $1 \mathrm{wt} . \%$ in a borosilicate glass in which it is associated with sodium ions.

Key words: Silicate melts / halogens / solubility / speciation / viscosity

\section{Introduction}

\subsection{Le verre}

Le verre est un solide amorphe formé par refroidissement rapide d'un liquide. Sa structure est désordonnée à longue distance mais il est organisé à plus faible distance [1]. Dans un verre d'oxydes, les éléments sous forme cationique s'associent à l'oxygène anionique $\left(\mathrm{O}^{2-}\right)$. Des polyèdres de coordination sont constitués par les éléments formateurs de réseau tels que le silicium, l'aluminium, le bore, le germanium ou le phosphore associés à des atomes d'oxygène en coordinence 3,4 ou 5 . Ces polyèdres (généralement triangles, tétraèdres ou pentaèdres) sont connectés par leurs sommets à des oxygènes que l'on désigne sous la terminologie d'oxygènes pontants [2] (Fig. 1). Dans un verre composé uniquement d'éléments formateurs de réseau, la structure est entièrement polymérisée. Cependant, les principaux éléments ayant la capacité de former seuls des verres sont $\mathrm{Si}$ dans $\mathrm{SiO}_{2}, \mathrm{Ge}$ dans $\mathrm{GeO}_{2}$ et $\mathrm{B}$ dans $\mathrm{B}_{2} \mathrm{O}_{3}$. D'autres éléments tels que les alcalins, les alcalino-terreux ou les métaux de transitions sont des éléments modificateurs de réseau qui vont casser les liaisons covalentes entre deux formateurs de réseau. Par exemple, l'ajout de $\mathrm{Na}_{2} \mathrm{O}$ dans un verre de silice crée des oxygènes non-pontants et dépolymérise le réseau. La viscosité de ces verres est très inférieure à celle du verre de silice [3]. Pour certains éléments formateurs de réseau,

\footnotetext{
${ }^{1}$ Institut de Physique du Globe de Paris, Géomatériaux, CNRS-UMR7154, Sorbonne Paris Cité, 1 rue Jussieu, 75005 Paris, France

grousset@ipgp.fr

2 CEA, DAM, DIF, 91297 Arpajon, France
} 


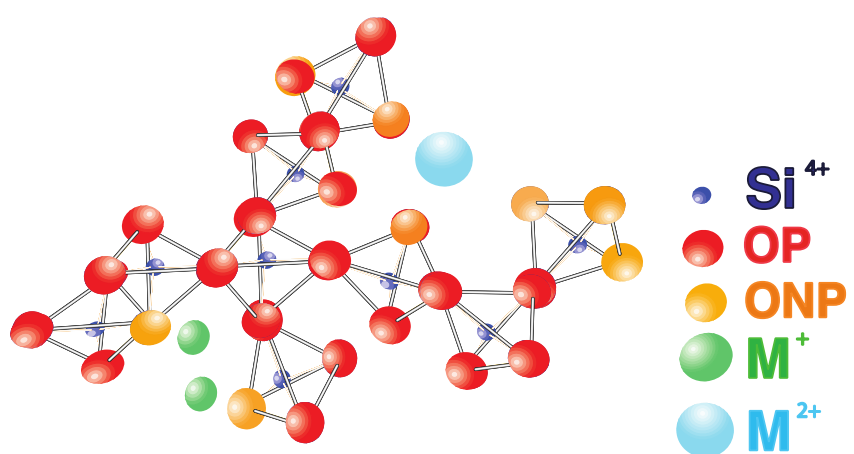

Fig. 1. Structure d'un réseau silicaté. Le silicium est au centre des tétraèdres. Les oxygènes pontants $(\mathrm{OP})$ sont en rouge, les oxygènes non pontants en orange $(\mathrm{ONP})$. Les cations modificateurs de réseau sont en vert et bleu $\left(\mathrm{M}^{+}\right.$et $\left.\mathrm{M}^{2+}\right)$. Les tailles des ions et les distances interatomiques ne sont pas respectées (d'après [50]).

Fig. 1. Structure of silicate glassy network. Silicon atoms are in the middle of tetrahedra. In red brindging oxygens (OP), in orange non-bridging oxygens (ONP), in green and blue network-modifiers $\left(\mathrm{M}^{+}\right.$and $\left.\mathrm{M}^{2+}\right)$. Ion sizes and interatomic distances are not respected (after [50]).

comme l'aluminium, la coordinence tétraédrique entraîne un défaut de charge qui est comblé par les éléments alcalins ou alcalino-terreux qui deviennent alors des compensateurs de charge. Malgré une absence d'ordre à grande échelle, les différents constituants du verre ne sont pas répartis de manière aléatoire. Le réseau silicaté est organisé en deux sous-réseaux : un domaine polymérisé composé par les formateurs de réseau connectés par les oxygènes pontant entrecoupé par des chenaux formés par les éléments modificateurs de réseau liés aux oxygènes non pontants [4-6].

Nous verrons que pour les halogènes, généralement présents sous forme anionique, la désignation comme formateur ou modificateur de réseau n'est pas aisée. Les termes de verre ou de silicate fondu sont utilisés ici comme synonyme car ils représentent une même composition chimique malgré leur différence d'état apparent (fortement visqueux pour l'un et liquide pour l'autre) essentiellement due à la température. Les expériences de solubilité, de diffusion et de viscosité sont faites dans un matériau fondu plus ou moins visqueux alors que les analyses chimiques et les mesures de spéciation sont effectuées sur un matériau solide vitreux.

\subsection{Les halogènes}

Le groupe des halogènes se compose du fluor $(\mathrm{F})$, du chlore $(\mathrm{Cl})$, du brome $(\mathrm{Br})$, de l'iode (I) et de l'astate (At). Le tableau 1 présente les principales caractéristiques de cette famille. Fortement électronégatifs, ce sont des éléments très réactifs qui forment volontiers des sels avec les métaux. Excepté le fluor qui n'en possède que deux ( - I et 0$)$, les halogènes présentent de nombreux états d'oxydation de - I à + VII. Ils se combinent facilement avec un électron pour former des ions halogénures $\left(\mathrm{X}^{-}\right)$, état d'oxydation le plus répandu dans la nature. Dans la suite de cette revue, l'astate ne sera pas considéré. En effet, cet élément possède seulement des isotopes radioactifs à très courte période, ce pourquoi il a très peu été étudié.

En sciences de la Terre, les halogènes sont étudiés principalement dans des verres issus de roches aluminosilicatées. Ces éléments sont volatils et sont issus des gaz volcaniques aux côtés de l'eau, du dioxyde de carbone et des espèces soufrées. Éléments mineurs ou en trace, ils ont un comportement incompatible (fractionnés en faveur du liquide silicaté pendant la cristallisation) et sont préférentiellement incorporés dans les fluides aqueux (excepté le fluor). Ces caractéristiques en font des traceurs géochimiques pour comprendre certains processus géodynamiques. Par exemple, la déshydratation de la croûte terrestre lors d'un processus de subduction peut être suivi grâce aux halogènes [7].

Les verres d'halogénures et d'oxyhalogénures (principalement des verres de fluorures ou oxyfluorés) sont synthétisés pour leurs propriétés optiques [8-10]. Dans ces verres, le fluor est un élément majeur de la composition chimique, incorporé dès la fabrication du verre. Les études se concentrent sur les propriétés optiques de ces matériaux (généralement dopés en terres rares [11]) et n'abordent pas la solubilité du fluor ou son effet sur la structure du verre. Ces compositions ne seront pas développées ici.

Les déchets nucléaires de haute activité (dont les produits de fission radioactifs) sont aujourd'hui stockés dans des matrices vitreuses. Les contraintes d'élaboration mais aussi d'entreposage à long terme de ces verres nucléaires ont conduit à considérer différentes compositions chimiques. Les halogènes font partie de ces déchets nucléaires et l'enjeu principal est de stocker de manière pérenne l'isotope radioactif ${ }^{129} \mathrm{I}$ de demivie 15,7 Ma [12]. Une approche consiste à l'incorporer sous forme AgI. Par exemple, un verre de phosphate peut incorporer jusqu'à $28 \%$ molaire d'iodure d'argent (AgI) [13]. L'incorporation d'iode sous forme $\mathrm{I}_{2}$ semble plus complexe, notamment à cause de sa volatilité [14]. Les borosilicates, dont les propriétés permettent d'incorporer beaucoup de produits de fission et de résister aux radiations, sont aussi de bons candidats pour ce type d'application [15-17].

L'étude de l'incorporation des halogènes et de leur rôle structural dans les verres silicatés est indispensable pour comprendre leurs implications dans les différents domaines présentés précédemment. Cet article propose une synthèse sur la solubilité, la spéciation et la diffusion des halogènes dans les silicates fondus ainsi que leur effet sur la viscosité de ces compositions.

\section{Solubilité}

La solubilité des halogènes dans un verre silicaté peut être déterminée à partir du mélange d'un silicate fondu et d'un sel d'halogénure (e.g. [18]), par solubilisation de l'halogène gazeux à la surface du verre (e.g. [19]) ou encore par équilibration d'un silicate fondu et d'un fluide aqueux enrichi en halogènes (e.g. [20]). Cette dernière 
Tableau 1. Caractéristiques des halogènes.

Table 1. Properties of the halogens.

\begin{tabular}{|c|c|c|c|c|}
\hline Élément & Fluor $(\mathrm{F})$ & Chlore (Cl) & Brome $(\mathrm{Br})$ & Iode (I) \\
\hline Numéro atomique & 9 & 17 & 35 & 53 \\
\hline Masse atomique & 19,00 & 35,45 & 79,90 & 126,91 \\
\hline $\begin{array}{l}\text { Électronégativité } \\
\text { de Pauling }\end{array}$ & 3,98 & 3,16 & 2,96 & 2,66 \\
\hline Valence & 1 & $1,3,5,7$ & $1,3,5,7$ & $1,3,5,7$ \\
\hline Etats d'oxydation & $-\mathrm{I}, 0$ & $-\mathrm{I}, 0,+\mathrm{I},+\mathrm{III},+\mathrm{V},+\mathrm{VII}$ & $-\mathrm{I}, 0,+\mathrm{I},+\mathrm{III},+\mathrm{V},+\mathrm{VII}$ & $-\mathrm{I}, 0,+\mathrm{I},+\mathrm{III},+\mathrm{V},+\mathrm{VII}$ \\
\hline Rayon covalent $(\mathrm{pm})^{1}$ & 71 & 99 & 114 & 133 \\
\hline $\begin{array}{l}\text { Rayon de van der } \\
\text { Waals }(\mathrm{pm})^{2}\end{array}$ & 147 & 175 & 185 & 198 \\
\hline Rayon ionique $(\mathrm{pm})^{3}$ & ${ }^{[6]} \mathrm{F}^{-}: 133$ & $\begin{array}{c}{ }^{[6]} \mathrm{Cl}^{-}: 181 \\
{ }^{[3 \mathrm{py}]} \mathrm{Cl}^{5+}: 12 \\
{ }^{[4]} \mathrm{Cl}^{7+}: 8\end{array}$ & $\begin{array}{c}{ }^{[6]} \mathrm{Br}^{-}: 196 \\
{ }^{[3 p y]} \mathrm{Br}^{5+}: 31 \\
{ }^{[4]} \mathrm{Br}^{7+}: 25 \\
{ }^{[6]} \mathrm{Br}^{7+}: 39\end{array}$ & $\begin{array}{c}{ }^{[6]} \mathrm{I}^{-}: 220 \\
{ }^{[3 \mathrm{py}]} \mathrm{I}^{5+}: 44 \\
{ }^{[6]} \mathrm{I}^{5+}: 95 \\
{ }^{[4]} \mathrm{I}^{7+}: 42 \\
{ }^{[6]} \mathrm{I}^{7+}: 53\end{array}$ \\
\hline
\end{tabular}

${ }^{1}$ Rayon covalent : taille d'un atome engagé dans une liaison covalente.

${ }^{2}$ Rayon de van der Waals : rayon atomique modélisant la distance adéquate entre 2 atomes non covalents.

3 Rayon ionique en fonction de la coordinence des ions (entre crochets) et de la valence : taille de l'ion dans un réseau cristallisé. [3py] : configuration pyramidale.

Origine des données : [60].

technique permet notamment de calculer le coefficient de partage entre le silicate fondu et un fluide hydraté. Ces expériences montrent la saturation en halogènes du silicate vitreux dans les conditions de pression et de température des expériences. La solubilité correspond alors à la concentration maximale de l'élément dans le verre. Le tableau 2 présente la solubilité des différents halogènes en fonction de la composition chimique du silicate fondu étudié. La colonne « concentration » du tableau montre l'intervalle des teneurs en halogènes obtenu pour chaque expérience.

L'influence de la pression et de la température sur la solubilité des halogènes n'est pas très bien comprise et n'a pas été étudiée dans le détail pour une même composition chimique. Lorsqu'il est possible de comparer les résultats, pour une même température et une même composition chimique (par exemple pour le brome), l'effet de la pression n'est pas généralisable. Parfois l'augmentation de pression favorise la solubilité, parfois non [20]. Par ailleurs, il semble que la solubilité du chlore dans un liquide granitique soit indépendante de la pression [21]. L'influence de la température sur la solubilité des halogènes n'est pas mieux comprise que celle de la pression. Pour les études où la comparaison est possible, les auteurs indiquent que la température ne semble pas avoir une influence majeure sur la solubilité [20,21] et les écarts de température des expériences sont généralement faibles (Tab. 2).

Pour tous les halogènes, la solubilité dans un silicate vitreux dépend fortement de la composition chimique. Néanmoins, pour une même composition chimique, la solubilité décroît avec la taille de l'halogène. En effet, la concentration maximale d'iode dans un liquide de composition albitique est quatre fois moins élevée que celle du brome, elle-même deux fois moins élevée que celle du chlore (Tab. 2). On peut comprendre ce fait en considérant simplement les rayons atomiques des halogènes (Tab. 1). Plus l'atome sera gros et plus il sera difficile de l'incorporer dans un matériau vitreux ou cristallin.

Le fluor est l'halogène le plus soluble dans les silicates fondus (Tab. 2). Sa concentration peut atteindre jusqu'à $16 \%$ massique dans un liquide à $50 \%$ molaire de silice. La solubilité du fluor diminue avec l'augmentation de la proportion de silice [22]. Contrairement aux autres halogènes, le fluor est plus soluble dans les silicates fondus que dans les fluides aqueux. C'est notamment le cas dans une composition rhyolitique [21]. Cependant, dans une phonolite hydratée, le coefficient de partage du fluor est en faveur du fluide aqueux [23]. Cela explique la faible solubilité du fluor (jusqu'à 1,3\% massique) dans une phonolite hydratée, comparable à celle du chlore $(0,7 \%$ massique). Ainsi le comportement du fluor dépend fortement de la composition chimique étudiée et notamment du degré de polymérisation du système (une rhyolite étant plus polymérisée qu'une phonolite) [23].

La solubilité du chlore varie selon la composition du verre mais sa saturation est atteinte pour des concentrations plus basses que dans le cas du fluor (Tab. 2). La concentration maximale $(2,5 \%)$ est obtenue dans un basalte [21]. La proportion de silice semble jouer un rôle dans son incorporation dans une matrice vitreuse. En effet, un liquide basaltique contenant $50 \%$ massique de $\mathrm{SiO}_{2}$ incorporera $1,45 \%$ massique de chlore alors qu'une andésite, à $60 \%$ de $\mathrm{SiO}_{2}$, n'en assimilera que $0,4 \%$ [24]. Cela peut révéler la corrélation inverse entre la polymérisation du réseau et la solubilité d'un élément dans un silicate vitreux. Néanmoins, ces expériences ont été réalisées à la même température. Or la viscosité de l'andésite est plus importante que celle du basalte à la même température. 
Tableau 2. Solubilité* des halogènes dans divers liquides silicatés.

Table 2. Solubility* of the halogens in various silicate melts.

\begin{tabular}{lccccc}
\hline Élément & Liquide silicaté $^{\text {Concentration }}$ & $\begin{array}{c}\text { Température } \\
(\% \text { massique })^{*}\end{array}$ & $\begin{array}{c}\text { Pression } \\
\left({ }^{\circ} \mathrm{C}\right)\end{array}$ & Références \\
\hline $\mathrm{F}$ & $\mathrm{MeF}_{2}-\mathrm{MeO}^{-\mathrm{SiO}_{2}^{\mathrm{a}}}$ & $10-15$ & $1450-1565$ & 1 & {$[61]$} \\
$\mathrm{F}$ & ${\mathrm{CaO}-\mathrm{CaF}_{2}-\mathrm{SiO}_{2}}^{\mathrm{a}}$ & $3,8-8,4$ & $1550-1600$ & & {$[62]$} \\
$\mathrm{F}$ & $\mathrm{SiO}_{2}-\mathrm{Al}_{2} \mathrm{O}_{3}-\mathrm{MeF}_{2}^{\mathrm{a}}$ & $8-16$ & $1360-1640$ & 1 & {$[63]$} \\
$\mathrm{F}$ & phonolite hydratée $^{\mathrm{b}}$ & $0,1-1,3$ & $845-1000$ & & {$[23]$} \\
$\mathrm{Cl}$ & albite $^{\mathrm{c}}$ & $0,0131-0,1433$ & 900 & 2000 & {$[26]$} \\
$\mathrm{Cl}$ & basalte $^{\mathrm{d}}$ & $0,1-2,5$ & & & {$[21]$} \\
$\mathrm{Cl}$ & basalte $^{\mathrm{d}}$ & $<1,45$ & 1200 & 1 & {$[24]$} \\
$\mathrm{Cl}$ & andésite $_{\mathrm{e}}^{\mathrm{e}}$ & $<0,4$ & 1200 & 1 & {$[21]$} \\
$\mathrm{Cl}$ & granite anhydre $^{\mathrm{f}}$ & $0,5-1,5$ & $1100-1200$ & $1-2000$ & {$[21]$} \\
$\mathrm{Cl}$ & phonolite hydratée $^{\mathrm{b}}$ & $0,3-0,7$ & $845-1000$ & & {$[23]$} \\
$\mathrm{Br}$ & albite $^{\mathrm{c}}$ & $0,0005-0,0888$ & 900 & 2000 & {$[26]$} \\
$\mathrm{Br}$ & albite $^{\mathrm{c}}$ & $0,25-0,7853$ & $900-1080$ & $1000-2000$ & {$[20]$} \\
$\mathrm{Br}$ & haplogranite $^{\mathrm{f}}$ & $0,2804-0,3899$ & $900-1000$ & $1000-2000$ & {$[17]$} \\
$\mathrm{Br}$ & rhyolite $^{\mathrm{g}}$ & $0,4304-0,5887$ & $900-1000$ & $1000-2000$ & {$[17]$} \\
$\mathrm{Br}$ & pantellerite $^{\mathrm{h}}$ & $0,9745-1,1250$ & $900-1000$ & $1000-2000$ & {$[17]$} \\
$\mathrm{I}$ & albite $^{\mathrm{c}}$ & $0,004-0,021$ & 900 & 2000 & {$[23]$} \\
$\mathrm{I}$ & basalte $^{\mathrm{d}}$ & $0,098-0,616$ & $1268-1300$ & $1,94-8,21$ & {$[19]$} \\
$\mathrm{I}$ & borosilicate $^{\mathrm{i}}$ & $0,01-1$ & 1000 & & {$[18]$} \\
\hline
\end{tabular}

* Concentrations reportées sous forme d'intervalle min-max des teneurs en halogènes obtenues pour chaque expérience.

${ }^{\text {a }} \mathrm{Me}=$ alcalino-terreux parmi $\mathrm{Mg}$, Sr et Ba.

Composition moyenne (wt \%) :

b phonolite hydratée : $57 \% \mathrm{SiO}_{2}, 20 \% \mathrm{Al}_{2} \mathrm{O}_{3}, 13 \% \mathrm{Na}_{2} \mathrm{O}+\mathrm{K}_{2} \mathrm{O}, 5 \% \mathrm{H}_{2} \mathrm{O}, 4 \%$ Fe total.

c albite : $66 \% \mathrm{SiO}_{2}, 18 \% \mathrm{Al}_{2} \mathrm{O}_{3}, 11 \% \mathrm{Na}_{2} \mathrm{O}, 3 \% \mathrm{H}_{2} \mathrm{O}$.

${ }^{\mathrm{d}}$ basalte : $50 \% \mathrm{SiO}_{2}, 15 \% \mathrm{Al}_{2} \mathrm{O}_{3}, 10 \% \mathrm{CaO}, 10 \%$ Fe total, $9 \% \mathrm{MgO}, 2,5 \% \mathrm{Na}_{2} \mathrm{O}+\mathrm{K}_{2} \mathrm{O}$.

e andésite : $59 \% \mathrm{SiO}_{2}, 17 \% \mathrm{Al}_{2} \mathrm{O}_{3}, 6 \% \mathrm{CaO}, 6 \%$ Fe total, $3 \% \mathrm{MgO}, 6,5 \% \mathrm{Na}_{2} \mathrm{O}+\mathrm{K}_{2} \mathrm{O}$.

f granite : $72 \% \mathrm{SiO}_{2}, 12 \% \mathrm{Al}_{2} \mathrm{O}_{3}, 9 \% \mathrm{Na}_{2} \mathrm{O}+\mathrm{K}_{2} \mathrm{O}$.

g rhyolite : $71 \% \mathrm{SiO}_{2}, 14 \% \mathrm{Al}_{2} \mathrm{O}_{3}, 8 \% \mathrm{Na}_{2} \mathrm{O}+\mathrm{K}_{2} \mathrm{O}, 2 \% \mathrm{CaO}$.

h pantellerite : $66 \% \mathrm{SiO}_{2}, 12 \% \mathrm{Al}_{2} \mathrm{O}_{3}, 15 \% \mathrm{Na}_{2} \mathrm{O}+\mathrm{K}_{2} \mathrm{O}$.

${ }^{\text {i }}$ borosilicate : $45 \% \mathrm{SiO}_{2}, 21 \% \mathrm{Na}_{2} \mathrm{O}, 10 \% \mathrm{~B} 2 \mathrm{O} 3,6 \% \mathrm{Al}_{2} \mathrm{O}_{3}, 5,5 \% \mathrm{Fe} 2 \mathrm{O} 3,3,5 \% \mathrm{ZnO}, 3 \% \mathrm{ZrO} 2,2 \% \mathrm{CaO}$.

Le rôle de la viscosité est donc aussi à prendre en compte. En effet, la viscosité étant reliée à la diffusivité des éléments composant le réseau silicaté [25], plus la viscosité est faible, plus la diffusivité est élevée. La solubilité du chlore dans l'andésite peut donc être sous-estimée du fait de la plus grande viscosité de cette dernière comparée au basalte. Pour une proportion comparable de silice et d'alumine, la solubilité du chlore augmente avec la proportion d'alcalins. La concentration maximale de chlore dans une phonolite (13\% d'alcalins) est de $0,7 \%$ alors qu'elle n'est que de 0,4\% dans une andésite $(6,5 \%$ d'alcalins) (Tab. 2). La solubilité du $\mathrm{Cl}$ dépend principalement des rapports $\left(\mathrm{M}_{2} \mathrm{O}+\mathrm{Al}_{2} \mathrm{O}_{3}\right) / \mathrm{SiO}_{2}$ et $\mathrm{M}_{2} \mathrm{O} / \mathrm{Al}_{2} \mathrm{O}_{3}$ dans le silicate fondu (M étant un alcalin). Elle est la plus basse lorsque le rapport $\mathrm{M}_{2} \mathrm{O} / \mathrm{Al}_{2} \mathrm{O}_{3}$ vaut 1 [21] ce qui correspond à un réseau vitreux totalement polymérisé.

En ce qui concerne le brome, son coefficient de partage entre un silicate fondu et un fluide aqueux est en faveur de ce dernier [26]. La solubilité du brome dans les silicates fondus est ainsi bien inférieure à celle du fluor. Les verres silicatés peuvent ainsi contenir jusqu'à 1,1\% massique de brome. La concentration maximale est obtenue pour une pantellerite, roche de composition granitique riche en alcalins et en silice (Tab. 2).
La solubilité de l'iode varie de 0,1 à $0,6 \%$ massique dans les liquides basaltiques (Tab. 2). Pour ces compositions, elle augmente avec la concentration croissante des formateurs de réseau $\left(\mathrm{SiO}_{2}\right.$ et $\left.\mathrm{Al}_{2} \mathrm{O}_{3}\right)$ et avec l'augmentation de la polymérisation du réseau silicaté [27]. La solubilité de l'iode atteint $1 \%$ massique dans un verre borosilicaté riche en sodium [18].

Ainsi, on peut retenir que la solubilité des halogènes dépend fortement de la composition du liquide mais aussi de l'élément lui-même. Le fluor est le plus soluble des halogènes dans les silicates et sa solubilité diminue avec la proportion de silice. La solubilité du chlore augmente avec la proportion en alcalins. La concentration en brome est maximale lorsque la composition est riche en alcalins. La solubilité de l'iode est comparable à celle du brome dans les liquides silicatés de compositions naturelles (basalte, albite). Elle est maximale pour un verre borosilicaté riche en alcalins synthétisé dans le cadre du confinement de déchets de faible activité.

\section{Spéciation}

L'étude de la structure fine du réseau silicaté permet de connaître l'environnement proche d'un élément 
donné et ainsi de déterminer la spéciation de cet élément au sein du verre. Deux techniques peuvent être utilisées pour déterminer la structure fine d'un verre silicaté : la spectroscopie d'absorption des rayons $\mathrm{X}$ et la résonance magnétique nucléaire (RMN). La spectroscopie d'absorption des rayons $\mathrm{X}$ est une méthode très utilisée et permet d'avoir accès à l'environnement local de l'atome visé. Le XANES (X-ray Absorption Near-Edge Spectrosopy) aux seuils $\mathrm{K}$ du chlore et de l'iode ( $\mathrm{K}$ pour iode ou L3) a notamment été utilisé pour déterminer la spéciation de ces halogènes et leurs plus proches voisins (e.g. [28, 29]). La RMN a été principalement utilisée pour déterminer le voisinage du fluor dans les verres (e.g. [30]).

La spéciation du fluor est particulière comparée à celle des autres halogènes. En effet, la similarité entre les rayons ioniques du fluor (133 pm) et de l'oxygène (132 pm) en fait un parfait candidat pour remplacer ce dernier dans les tétraèdres de coordination composant le réseau silicaté [31]. On peut ainsi imaginer que le fluor se lie aux formateurs de réseau tels que $\mathrm{Si}$ ou $\mathrm{Al}$. Alors que les liaisons $\mathrm{Si}-\mathrm{F}$ sont peu probables ou très peu abondantes [30,32], il a été montré que le fluor se lie à l'aluminium $[21,33,34]$. Ainsi, les structures $\mathrm{AlF}_{6}^{3-}$, $\mathrm{AlF}_{5}^{2-}$ ou $\mathrm{AlF}_{4}^{-}$peuvent être présentes dans des aluminosilicates de sodium riche en fluor [21]. La proportion de complexes Na-F décroît avec l'augmentation de la teneur en aluminium dans les systèmes $\mathrm{Na}_{2} \mathrm{O}-\mathrm{Al}_{2} \mathrm{O}_{3}$ $\mathrm{SiO}_{2}$ et $\mathrm{Na}_{2} \mathrm{O}-\mathrm{CaO}-\mathrm{Al}_{2} \mathrm{O}_{3}-\mathrm{SiO}_{2}$. Les compositions les plus riches en aluminium sont aussi les plus dépolymérisées par l'ajout du fluor. La spéciation du fluor dépend très fortement du ratio $\left[\mathrm{Na}_{2} \mathrm{O}+\mathrm{CaO}\right] / \mathrm{Al}_{2} \mathrm{O}_{3}$ [34]. La présence de fluor favorise l'apparition de complexes Al-F pentaou hexacoordonnés au détriment des espèce tétraédriques qui assurent la polymérisation du système [30,32]. Le fluor dépolymérise donc le réseau aluminosilicaté. Dans les systèmes $\mathrm{Na}_{2} \mathrm{O}-\mathrm{SiO}_{2}$ et $\mathrm{Na}_{2} \mathrm{O}-\mathrm{CaO}-\mathrm{SiO}_{2}$ (sans aluminium), le fluor se lie aux modificateurs de réseau ( $\mathrm{Na}$ et Ca) [33, 34].

En ce qui concerne le chlore, il s'associe avec les cations modificateurs de réseau tels que $\mathrm{Na}$ ou $\mathrm{Ca}$ dans les liquides silicatés et borosilicatés [28, 35]. Sa solubilité est d'ailleurs plus élevée dans les compositions riches en alcalins (Tab. 2). Néanmoins, dans le système NaO$\mathrm{CaO}-\mathrm{MgO}-\mathrm{Al}_{2} \mathrm{O}_{3}-\mathrm{SiO}_{2}$, le chlore s'allie préférentiellement aux cations divalents $\left(\mathrm{Ca}^{2+}\right.$ ou $\left.\mathrm{Mg}^{2+}\right)$ plutôt qu'aux alcalins [36].

Très récemment, une étude de la spéciation du brome dans des liquides silicatés hydratés a montré que le brome est entouré des ions sodium et que sa coordinence augmente avec la pression (passage de 3 à 6 ions sodium autour du brome) [37].

L'iode s'associe sous forme iodure avec les alcalins et principalement les ions sodium au sein du réseau vitreux $[18,29]$.

On peut ainsi retenir que la spéciation des halogènes dans le verre silicaté varie de façon spécifique avec l'élément considéré et la composition chimique du verre. Le fluor s'associe à l'aluminium à la place des oxygènes et dépolymérise le réseau. Il se lie aussi aux éléments mo- dificateurs du réseau (alcalins et alcalino-terreux) comme le chlore et l'iode. Le chlore s'associe plutôt avec les cations divalents alors que le brome et l'iode se combinent préférentiellement aux ions alcalins monovalents.

\section{Viscosité}

La figure 2 montre l'influence des halogènes ( $\mathrm{F}$ et $\mathrm{Cl}$ ) sur la viscosité des silicates fondus. La nomenclature peralumineux et peralcalin fait référence à la proportion relative d'éléments alcalins par rapport à l'aluminium dans la composition chimique exprimée par le rapport molaire $R=\left(\mathrm{Na}_{2} \mathrm{O}+\mathrm{CaO}+\mathrm{K}_{2} \mathrm{O}\right) / \mathrm{Al}_{2} \mathrm{O}_{3}$. Ainsi pour $R>0,5$, la composition est peralcaline et pour $R<0,5$, la composition est peralumineuse. Actuellement, aucune publication ne rapporte l'influence du brome ou de l'iode sur la viscosité des silicates vitreux ou fondus.

Quelle que soit la composition chimique étudiée, l'ajout de fluor provoque une baisse significative (au moins un ordre de grandeur) de la viscosité, y compris pour des compositions rhyolitiques (habituellement très visqueuses) [38-42]. Cette diminution de la viscosité s'explique par les changements structuraux du réseau silicaté impliqués par l'ajout du fluor. Nous avons vu que le fluor se lie à l'aluminium et favorise les espèces $\mathrm{Al}-\mathrm{F}$ penta- et hexa-coordonnées. Cela conduit à la dépolymérisation du réseau qui se traduit macroscopiquement par une baisse de la viscosité. Cette explication tient pour les systèmes aluminosilicatés. Le rôle structural du fluor dans un silicate sans aluminium n'est pas encore complètement compris [41]. En effet, le fluor baisse aussi la viscosité des systèmes $\mathrm{Na}_{2} \mathrm{O}-2 \mathrm{SiO}_{2}$ (NS2) et $\mathrm{Na}_{2} \mathrm{O}-\mathrm{CaO}-\mathrm{SiO}_{2}$ (NCS) qui ne comportent pas d'aluminium (Fig. 2). Cet halogène se combine également avec les éléments modificateurs de réseau. Cependant, l'association Na-F ou Ca-F devrait favoriser la proportion d'oxygènes pontants entraînant la polymérisation du réseau et une augmentation de la viscosité mais de telles expériences ne sont pas faciles et nécessiterait de coupler analyses structurales à haute température et mesure de viscosité à basse et haute température.

L'influence du chlore sur la viscosité des verres silicatés est moins visible et plus complexe que celle du fluor. Pour le système $\mathrm{Na}_{2} \mathrm{O}-2 \mathrm{SiO}_{2}-\mathrm{Fe}$ (NS2Fe), l'ajout de chlore provoque une légère diminution de la viscosité pour une température inférieure à $1100{ }^{\circ} \mathrm{C}$ et une augmentation pour une température supérieure à $1300{ }^{\circ} \mathrm{C}$ [39]. Il semble que cet effet soit aussi présent pour le système $\mathrm{Na}_{2} \mathrm{O}-\mathrm{FeO}-\mathrm{Al}_{2} \mathrm{O}_{3}-\mathrm{SiO}_{2}$ (NFeAS) peralumineux même si les courbes de viscosité sont très proches [40] (Fig. 2). Dans ce système, le chlore s'associant au cation divalent (ici $\mathrm{Fe}^{2+}$, dont la proportion varie avec la température), la formation de tri-clusters $\left(\mathrm{Al}^{3+}, \mathrm{Fe}^{3+}\right) \mathrm{Si}_{2} \mathrm{O}_{5}$ (dans lesquels les atomes $\mathrm{Al}$ et $\mathrm{Si}$ partagent une liaison covalente et ne sont plus reliés par un oxygène pontant) serait favorisée et influencerait la viscosité du verre enrichi en chlore [40]. La présence de ce type de tri-clusters n'a cependant pas été démontrée dans ces compositions. Des tri-clusters dans lesquels un atome d'oxygène assure le lien entre 
L. Grousset et al. : Matériaux \& Techniques 103, 405 (2015)

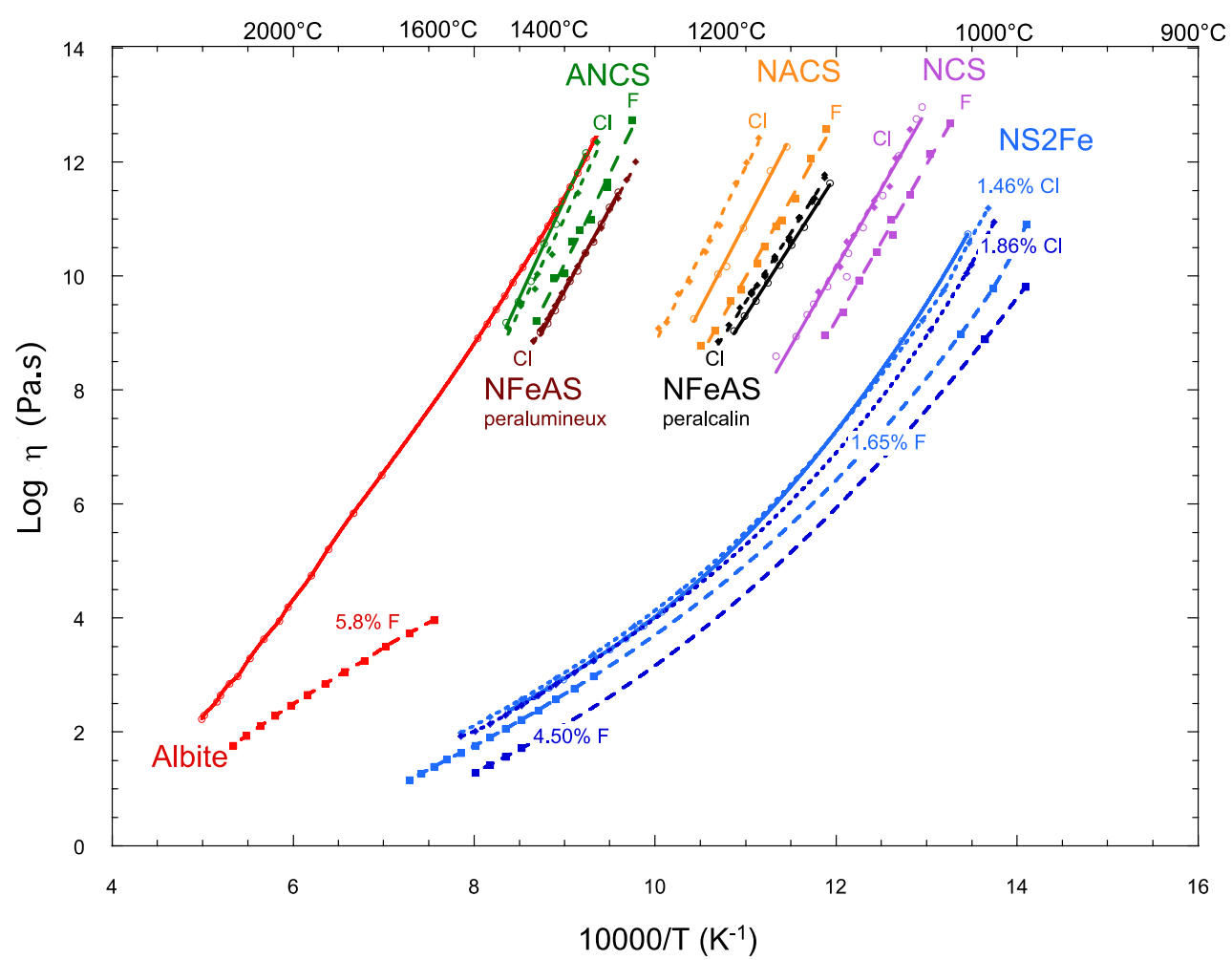

Fig. 2. Influence des halogènes $(\mathrm{F}$ et $\mathrm{Cl})$ sur la viscosité d'un verre silicaté. Trait plein : verres sans halogènes, tireté : verres avec fluor, pointillé : verre avec chlore. Données : albite [45,51], albite 5,8 \% F [38], système $\mathrm{Na}_{2} \mathrm{O}-\mathrm{SiO}$ - $\mathrm{FeO}$ ( $\mathrm{NS} 2 \mathrm{Fe}$ ) [39], système $\mathrm{Na}_{2} \mathrm{O}-\mathrm{Al}_{2} \mathrm{O}_{3}-\mathrm{CaO}-\mathrm{SiO}_{2}$ peralcalin (NACS), peralumineux (ANCS) et sans aluminium $(\mathrm{NCS})$ [41], système Na 2 -FeO$\mathrm{Al}_{2} \mathrm{O}_{3}-\mathrm{SiO}_{2}$ (NFeAS peralcalin et peralumineux) [40].

Fig. 2. Halogens ( $F$ and $C l$ ) effect on the viscosity of silicate glasses. Solid line: glasses without halogens, dashed line: F-bearing glasses, dotted line: Cl-bearing glasses. Data sources: albite [45, 51], albite 5,8\% F [38], $\mathrm{Na}_{2} \mathrm{O}-\mathrm{SiO} \mathrm{O}_{2}-\mathrm{FeO}$ ( $\mathrm{NS2Fe)} \mathrm{system} \mathrm{[39],}$ $\mathrm{Na}_{2} \mathrm{O}-\mathrm{Al}_{2} \mathrm{O}_{3}-\mathrm{CaO}-\mathrm{SiO}_{2}$ peralkalin ( $\mathrm{NACS}$ ), peraluminous (ANCS) and without aluminium ( $\mathrm{NCS}$ ) systems [41], $\mathrm{Na}_{2} \mathrm{O}-\mathrm{FeO}$ $\mathrm{Al}_{2} \mathrm{O}_{3}-\mathrm{SiO}_{2}$ (NFeAS peralkalin et peraluminous) system [40].

trois tétraèdres de coordination pourraient néanmoins être présents dans des compositions chimiques peralumineuses [43] et ont une signature spécifique en RMN [44] mais sont généralement présent en très faible quantité dans les verres aluminosilicatés [3,45]. Aucune influence du chlore n'est visible pour le système NCS. Dans le cas des systèmes peralcalins ( $\mathrm{NFeAS}$ et $\mathrm{Na}_{2} \mathrm{O}-\mathrm{Al}_{2} \mathrm{O}_{3}-\mathrm{CaO}-$ $\mathrm{SiO}_{2}$ (NACS)), l'ajout de chlore augmente la viscosité du silicate fondu. Nous avons vu que le chlore s'associe aux éléments $\mathrm{Na}$ et $\mathrm{Ca}[28]$ pour former des oxygènes pontants en se combinant avec les modificateurs de réseau. Cela provoque une augmentation de la polymérisation du réseau et de la viscosité $[40,41]$. Pour le système peralumineux $\mathrm{Al}_{2} \mathrm{O}_{3}-\mathrm{Na}_{2} \mathrm{O}-\mathrm{CaO}-\mathrm{SiO}_{2}$ (ANCS), le chlore semble abaisser la viscosité. Cette tendance est néanmoins très peu visible, les viscosités du verre initial et de celui enrichi en chlore étant très proches (Fig. 2). Dans ce système, tous les éléments alcalins et alcalino-terreux sont mobilisés pour compenser les charges des tétraèdres d'aluminium. Le chlore s'associant aux compensateurs de charges $\left(\mathrm{Ca}^{2+}\right.$ ou $\left.\mathrm{Na}^{+}\right)$, c'est l'aluminium lui-même (sous forme $\mathrm{Al}^{3+}$ ) qui assurerait le rôle de compensateur de charge [41]. Cependant, rien de laisse supposer que l'aluminium puisse se trouver sous forme ionique isolée (à la manière d'un ion sodium ou calcium) au sein du réseau vitreux. En plus des tétraèdres, un système peralumineux présente des aluminiums penta- ou hexacoordonnés. Il a été montré que l'aluminium pentacoordonné favorise la polymérisation du réseau silicaté [3]. Il semble donc que l'effet du chlore sur la viscosité d'un système peralumineux soit plus complexe que dans un système peralcalin.

Ainsi, on peut retenir que l'influence du fluor et du chlore sur la viscosité des silicates est contrastée. Alors que le fluor abaisse systématiquement la viscosité, la présence de chlore a plutôt tendance à l'augmenter dans les systèmes peralcalins. Les explications liées à la spéciation sont convaincantes pour le fluor dans les aluminosilicates et pour le chlore dans les systèmes peralcalins. Néanmoins, le rôle de ces halogènes n'est pas bien défini pour d'autres systèmes.

\section{Diffusion}

Peu d'études présentent des coefficients de diffusion des halogènes dans les silicates fondus. La figure 3 présente les diffusivités du fluor, du chlore et du brome 


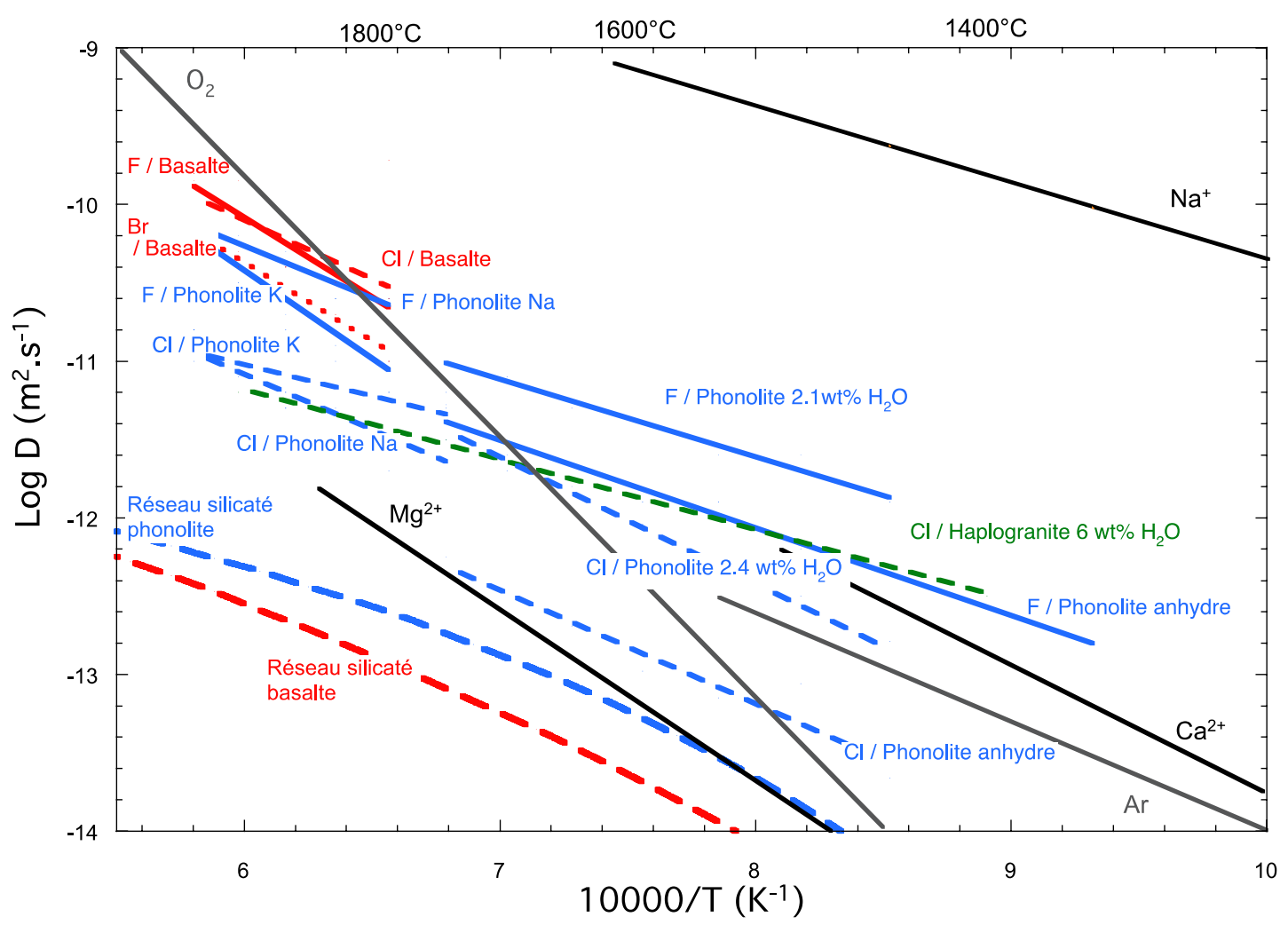

Fig. 3. Coefficient de diffusion en fonction de la température de $\mathrm{F}, \mathrm{Cl}$ et $\mathrm{Br}$; origine des données : basalte [47], Na et K-phonolite [46], Phonolite anhydre et hydratée [52], Cl Haplogranite hydratée [53], $\mathrm{O}_{2}$ [54], $\mathrm{Na}^{+}$dans une obsidienne [55], $\mathrm{Ca}^{2+}$ et $\mathrm{Mg}^{2+}$ dans une albite ou une jadéite [56,57], Ar dans une albite [58], réseau silicaté d'un basalte [59], réseau silicaté d'une phonolite [données non publiées].

Fig. 3. Diffusion coefficient of halogens (F, Cl and Br) as a function of temperature. Data sources: basalt [47], Na et K-phonolite [46], Anhydrous and hydrous phonolite [52], Cl-bearing hydrous haplogranite [53], $\mathrm{O}_{2}$ [54], $\mathrm{Na}^{+}$in obsidian [55], $\mathrm{Ca}^{2+}$ et $\mathrm{Mg}^{2+}$ in albite or jadeite [56,57], Ar in albite [58], basalt silicate network [59], phonolite silicate network [unpublished data].

dans trois types de silicates (un basalte, une phonolite et un haplogranite avec respectivement $50 \%, 57 \%$ et $70 \%$ de silice). L'évolution de la diffusion en fonction de la température peut être modélisée par la loi d'Arrhenius [46]. Les coefficients de diffusion des éléments halogènes sont plus faibles pour un silicate plus riche en silice. La diffusion du fluor, par exemple, est plus lente dans une phonolite que dans un basalte. La diffusivité dépend aussi du rayon ionique de l'élément. Ainsi, dans une phonolite comme dans un basalte, les coefficients de diffusion diminuent avec la taille de l'élément $\left(D_{\mathrm{F}}>D_{\mathrm{Cl}}>D_{\mathrm{Br}}\right)[46,47]$ (Fig. 3). Aucune donnée ne renseigne sur la diffusion de l'iode au sein d'un réseau silicaté.

Pour comparaison, sur la figure 3 , sont aussi reportés les diffusivités de certains cations $\left(\mathrm{Na}^{+}, \mathrm{Ca}^{2+}, \mathrm{Mg}^{2+}\right)$, de certains gaz $\left(\mathrm{O}_{2}\right.$ et $\left.\mathrm{Ar}\right)$ et du réseau silicaté d'un basalte et d'une phonolite. Sous forme halogénures, les éléments halogènes ont un rayon ionique important (Tab. 1), plus élevé que les alcalins comme par exemple le sodium (102 pm). Cette différence est encore plus marquée avec les cations divalents $\left(100 \mathrm{pm}\right.$ pour $\mathrm{Ca}^{2+}$ et $72 \mathrm{pm}$ pour $\left.\mathrm{Mg}^{2+}\right)$. Pourtant, le fluor et le chlore ont des coefficients de diffusion comparables à ceux des cations divalents (Fig. 3). Cela pourrait traduire la spéciation du fluor et du chlore. Nous avons vu que ces halogènes s'associaient aux alcalino-terreux au sein du réseau silicaté (en particulier le chlore). Le fluor et le chlore migreraient alors à une vitesse comparable à ces éléments au sein des chenaux formés par les modificateurs de réseau. Les coefficients de diffusion des halogènes sont également proches de celui de l'argon. Là encore, la différence de rayons est importante (71 pm pour Ar) mais cela pourrait refléter le fait que les halogènes migrent à travers le réseau silicaté à la manière des gaz rares, c'est à dire au sein du volume libre du verre [46] (Fig. 3).

\section{Discussion}

Dans les silicates fondus, le fluor a un comportement distinct des autres halogènes. Il est l'halogène le plus soluble dans les silicates. En se substituant à l'oxygène et en s'associant à l'aluminium, dont la coordinence passe de tétraédrique à penta- ou hexaédrique, il favorise la dépolymérisation du réseau qui se traduit par une baisse de la viscosité. Il participe ainsi à l'élaboration des 
polyèdres de coordination sans permettre leur association (contrairement à l'oxygène, il ne forme qu'une seule liaison covalente).

Le chlore est beaucoup moins soluble que le fluor mais sa solubilité est favorisée par la présence d'alcalins dans la composition du liquide. Il s'associe aux cations modificateurs de réseau comme le calcium ou le sodium. Son effet sur la viscosité est dépendant de la composition chimique, notamment de la proportion d'alcalins et d'aluminium. Du fait de l'association du chlore avec $\mathrm{Ca}$ ou $\mathrm{Na}$, un liquide peralcalin sera plus visqueux en présence de $\mathrm{Cl}$. L'influence du chlore sur un liquide peralumineux est plus complexe. La concentration du brome est favorisée dans un liquide riche en silice et peralcalin. Cet halogène se combine aux ions sodium aus sein du réseau vitreux. Peu d'études rapportent la solubilité de l'iode dans les silicates naturels mais une limite de solubilité de l'iode (jusqu'à $1 \%$ massique) a été établie dans un borosilicate sodique (verre de confinement des déchets nucléaires). Dans ce même verre, l'iode s'associe au sodium. Les effets du brome et de l'iode sur la viscosité des silicates fondus sont peu connus.

Dans les études traitant de leur solubilité, l'état d'oxydation des halogènes est très peu abordé. Les sels d'halogénures $\left(\mathrm{NaCl}, \mathrm{KI}, \mathrm{CaF}_{2} \ldots\right)$ sont majoritairement utilisés comme source d'halogènes pour les études de solubilité $[18,20,24]$ et les analyses chimiques (par microsonde électronique ou fluorescence des rayons X) ne permettent pas de déterminer leur degré d'oxydation. Du fait des expériences réalisées, les halogènes sont généralement admis sous leur état d'oxydation -I (c'est-à-dire halogénure) dans les verres silicatés. La solubilité de ces éléments n'a pas été étudiée selon l'état d'oxydation. Or la grande diversité du redox des halogènes (excepté celui du fluor) (Tab. 1) permet d'envisager plusieurs états d'oxydation d'un halogène dans un silicate fondu. Différents états de l'iode (majoritairement iodure $\left(\mathrm{I}^{-}\right)$ mais également iodate $\left(\mathrm{IO}_{3}^{-}\right)$et iode élémentaire $\left.\left(\mathrm{I}_{2}\right)\right)$ ont déjà été observés dans un verre borosilicaté [18,48]. L'état redox des halogènes dans les silicates peut avoir un impact sur la structure fine du verre. L'ion iodate se combine-t-il aux mêmes éléments que l'ion iodure? Le rayon ionique de l'iode sous ces deux formes est très différent (Tab. 1). L'état redox d'un halogène pourrait avoir une influence sur les autres éléments multivalents du verre comme le fer, dont le redox détermine par ailleurs son rôle structural au sein du réseau silicaté (e.g. [49]).

\section{Conclusion}

L'incorporation et le rôle structural des halogènes dans un silicate dépendent principalement de l'élément lui-même et de la composition chimique du verre. Le comportement du fluor est différent de celui des autres halogènes. Son rôle structural est très particulier car il s'associe à l'aluminium à la place de l'oxygène. Structuralement, les autres halogènes se situent plutôt au voisinage des cations modificateurs de réseau. Cela se traduit par une forte solubilité du fluor dans les aluminosilicates fondus et une solubilité du chlore ou du brome favorisée par la présence d'alcalins. Macroscopiquement, le fluor abaisse la viscosité de tous les silicates en dépolymérisant le réseau alors que le chlore augment la viscosité des liquides peralcalins. L'état redox des halogènes a été très peu étudié jusqu'à présent mais plusieurs états d'oxydation de l'iode ont été observés dans des borosilicates. L'état redox des halogènes pourrait être une clef pour la compréhension des mécanismes qui contrôlent leur solubilité, leur diffusion et la viscosité des silicates fondus.

\section{Références}

[1] R.H. Doremus, Glass Science, Wiley, New York, 1973

[2] W.H. Zachariasen, J. Am. Chem. Soc. 54 (1932) 3841-3851

[3] D.R. Neuville, P. Florian, C. Le Losq, D. Massiot, Matériaux \& Techniques 98 (2010) 395-402

[4] G.N. Greaves, A. Fontaine, P. Lagarde, D. Raoux, S.J. Gurman, Nature 293 (1981) 611-616

[5] G.N. Greaves, J. Non-Cryst. Solids 71 (1985) 203-217

[6] G.H. Frischat, J.F. Poggemann, G. Heide, J. Non-Cryst. Solids 345 (2004) 197-202

[7] M.A. Kendrick, J.D. Woodhead, V.S. Kamenetsky, Geology 40 (2012) 1075-1078

[8] M.J. Dejneka, MRS Bulletin 23 (1998) 57-62

[9] J.-L. Adam, J. Non-Cryst. Solids 287 (2001) 401-404

[10] V. Nazabal, M. Poulain, M. Olivier, P. Pirasteh, P. Camy, J.L. Doualan, S. Guy, T. Djouama, A. Boutarfaia, J.L. Adam, J. Fluorine Chem. 134 (2012) 18-23

[11] A. Biswas, G.S. Maciel, C.S. Friend, P.N. Prasad, J. NonCryst. Solids 316 (2003) 393-397

[12] P. Hrma, Report PNNL-19361, Pacific Northwest National Laboratory, Richmond, Washington (2010)

[13] T. Lemesle, F.O. Méar, L. Campayo, O. Pinet, B. Revel, L. Montagne, J. Hazardous Mater. 264 (2014) 117-126

[14] D.S.V. Perera, E.R. Trautman, R.L. Begg, B.D., Waste Management Conference, Feb 29-Mar 4, 2004, Tucson AZ, 2004

[15] H. Matzke, E. Vernaz, J. Nucl. Mater. 201 (1993) 295-309

[16] S.G. Luo, J.W. Sheng, B.L. Tang, J. Nucl. Mater. 298 (2001) 180-183

[17] S. Baccaro, N. Catallo, A. Cemmi, G. Sharma, Nucl. Instrum. Methods B 269 (2011) 167-173

[18] B.J. Riley, M.J. Schweiger, D.-S. Kim, W.W. Lukens Jr, B.D. Williams, C. Iovin, C.P. Rodriguez, N.R. Overman, M.E. Bowden, D.R. Dixon, J.V. Crum, J.S. McCloy, A.A. Kruger, J. Nucl. Mater. 452 (2014) 178-188

[19] D.S. Musselwhite, M.J. Drake, Icarus 148 (2000) 160-175

[20] H. Bureau, N. Metrich, Geochim. Cosmoch. Acta 67 (2003) 1689-1697

[21] M.R. Carroll, J.D. Webster, Volatiles in Magmas 30 (1994) 231-279

[22] B.O. Mysen, P. Richet, Silicate glasses and melts: Properties and structure, Elsevier, Amsterdam., 2005

[23] V.Y. Chevychelov, R.E. Botcharnikov, F. Hoitz, Chem. Geology 256 (2008) 172-184

[24] B. Iwasaki, T. Katsura, Bull. Chem. Soc. Jpn 40 (1967) 554

[25] H. Eyring, J. Chem. Phys. 4 (1936) 283

[26] H. Bureau, H. Keppler, N. Metrich, Earth Planetary Sci. Lett. 183 (2000) 51-60 
[27] D.S. Musselwhite, Planetary Sciences, University of Arizona, 1995

[28] D.A. McKeown, H. Gan, I.L. Pegg, W.C. Stolte, I.N. Demchenko, J. Nucl. Mater. 408 (2011) 236-245

[29] D.A. McKeown, I.S. Muller, I.L. Pegg, J. Nucl. Mater. 456 (2015) 182-191

[30] T. Schaller, D.B. Dingwell, H. Keppler, W. Knöller, L. Merwin, A. Sebald, Geochim. Cosmoch. Acta 56 (1992) 701-707

[31] A. Aiuppa, D.R. Baker, J.D. Webster, Chem. Geology 263 (2009) 1-18

[32] N.G. Karpukhina, U. Werner-Zwanziger, J.W. Zwanziger, A.A. Kiprianov, J. Phys. Chem. B 111 (2007) 10413-10420

[33] B.O. Mysen, G.D. Cody, A. Smith, Geochim. Cosmoch. Acta 68 (2004) 2745-2769

[34] A. Baasner, B.C. Schmidt, R. Dupree, S.L. Webb, Geochim. Cosmoch. Acta 132 (2014) 151-169

[35] T.O. Sandland, L.S. Du, F. Stebbins, J.D. Webster, Geochim. Cosmoch. Acta 68 (2004) 5059-5069

[36] K.A. Evans, J.A. Mavrogenes, H.S. O'Neill, N.S. Keller, L.Y. Jang, Geochem. Geophy. Geosyst. 9 (2008) 15

[37] B. Cochain, C. Sanloup, C. de Grouchy, C. Crépisson, H. Bureau, C. Leroy, I. Kantor, T. Irifune, Chem. Geology 404 (2015) 18-26

[38] D.B. Dingwell, B.O. Mysen, Earth Planetary Sci. Lett. 74 (1985) 266-274

[39] D.B. Dingwell, K.U. Hess, Am. Miner. 83 (1998) 1016-1021

[40] M. Zimova, S. Webb, Am. Miner. 91 (2006) 344-352

[41] A. Baasner, B.C. Schmidt, S.L. Webb, Chem. Geology 346 (2013) 172-183

[42] E.H. Christiansen, D.M. Burt, M.F. Sheridan, R.T. Wilson, Contr. Mineral. Petrol. 83 (1983) 16-30

[43] E.D. Lacy, Phys. Chem. Glasses 4 (1963) 234-238

[44] D. Iuga, C. Morais, Z.H. Gan, D.R. Neuville, L. Cormier, D. Massiot, J. Am. Chem. Soc. 127 (2005) 11540-11541
[45] C. Le Losq, D.R. Neuville, P. Florian, G.S. Henderson, D. Massiot, Geochim. Cosmoch. Acta 126 (2014) 495-517

[46] H. Balcone-Boissard, D.R. Baker, B. Villemant, G. Boudon, Chem. Geology 263 (2009) 89-98

[47] M. Alletti, D.R. Baker, C. Freda, Geochim. Cosmochi. Acta 71 (2007) 3570-3580

[48] L. Grousset, D.R. Neuville, E. Pili, Goldschmidt, Sacramento, 2014, p. 864

[49] B.O. Mysen, Geochim. Cosmoch. Acta 70 (2006) 2337-2353

[50] C. Le Losq, Université Paris Diderot, 2012

[51] G. Urbain, Y. Bottinga, P. Richet, Geochim. Cosmoch. Acta 46 (1982) 1061-1072

[52] A. Böhm, B.C. Schmidt, Chem. Geology 346 (2013) 162-171

[53] T.B. Bai, A.F.K. van Groos, Geochim. Cosmoch. Acta 58 (1994) 113-123

[54] G. Oishi, R. Terai, H. Ueda, Oxygen diffusion in liquid silicates and relation to their viscosity, in: A. Cooper, A. Heuer (Eds.), Mass Transport Phenomena in Ceramics, Plenum Press, New York, 1975, pp. 297-310

[55] A. Jambon, J.P. Carron, Geochim. Cosmoch. Acta 40 (1976) 897-903

[56] A. Jambon, M.P. Semet, Earth Planetary Sci. Lett. 37 (1978) 445-450

[57] K. Roselieb, A. Jambon, Geochim. Cosmoch. Acta 66 (2002) 109-123

[58] K. Roselieb, W. Rammensee, H. Buttner, M. Rosenhauer, Chem. Geology 120 (1995) 1-13

[59] N. Villeneuve, D.R. Neuville, P. Boivin, P. Bachèlery, P. Richet, Chem. Geology 256 (2008) 242-251

[60] CRC Handbook of Chemistry and Physics, Internet Version 2005, CRC Press, Boca Raton, FL, 2005

[61] Z. Ershova, Y. Olshanskii, Geochemistry 3 (1957) 257-266

[62] R. Luth, Am. Miner. 73 (1988) 306-312

[63] Z. Ershova, Geochemistry 4 (1957) 350-358 